\title{
ARTICLE
}

\section{The therapeutic effects of ablative neurosurgical procedures on the spinal cord for intractable spinal spasticity}

\author{
Bunpot Sitthinamsuwan ${ }^{1}$, Pornchai Khumsawat ${ }^{1}$, Luckchai Phonwijit ${ }^{1}$, Sarun Nunta-aree ${ }^{1}$, Akkapong Nitising ${ }^{1}$ \\ and Sirilak Suksompong ${ }^{2}$
}

STUDY DESIGN: This research is a retrospective study.

OBJECTIVE: To study the therapeutic effects of operative procedures, including dorsal longitudinal myelotomy (DLM) and dorsal root entry zone lesion (DREZL) on spasticity and associated aspects.

SETTING: Tertiary university hospital in Bangkok, Thailand.

METHODS: Eighteen patients with refractory spasticity of spinal origin who underwent the operations were recruited. Clinical parameters for evaluating severity of spasticity and ambulatory status were compared between before and after surgery, and between surgeries. RESULTS: A statistically significant reduction of spasticity as measured by the Modified Ashworth Scale (MAS), Adductor Tone Rating Scale (ATRS) and Penn Spasm Frequency Scale (PSFS) was found after surgeries and in the overall analysis $(p<0.05)$. Chronic pressure ulcers disappeared postoperatively in 11 cases. All of 7 bed-ridden subjects experienced improvement in their ambulatory status postoperatively. DLM was found to be more effective than DREZL in reduction of spasticity.

CONCLUSION: Ablative neurosurgery on the spinal cord is still valuable in situations when intrathecal baclofen is unavailable. These operations are potentially effective in the treatment of intractable spasticity of spinal origin.

Spinal Cord Series and Cases (2017) 3, 17033; doi:10.1038/scsandc.2017.33; published online 8 June 2017

\section{INTRODUCTION}

Spasticity is a distinctive clinical phenomenon that occurs after damage to the spinal cord. It interferes with voluntary motor function, increases difficulty in self-care and caregiving, causes agonizing pain, musculoskeletal deformity, poor body posture, pressure ulcers and depression. ${ }^{1-4}$ Patients suffering from intractable harmful spasticity can gain benefit from surgical treatment. ${ }^{5}$

Intrathecal baclofen therapy (ITB) has become popular worldwide in the treatment of refractory widespread or regional spasticity. Its efficacy in reduction of spasticity has been proven. ${ }^{6-10}$ However, mechanical complications, drug overdose and withdrawal, and relatively high cost are major drawbacks of ITB.,11-13 In our country, intrathecal baclofen is not generally available and the costs of the hardware and drug are not covered by our national health insurance system. Therefore, we use ablative neurosurgical procedures instead of ITB for treating diffuse spasticity. These procedures consist of dorsal longitudinal myelotomy (DLM) (Figures 1 and 2) and dorsal root entry zone lesion (DREZL) (Figure 3 ) by using microsurgical techniques on the spinal cord.

The present study reports the therapeutic effects of these lesioning procedures on spasticity and related perspectives.

\section{MATERIALS AND METHODS}

Patient population

Eighteen patients from our institute who underwent DLM and DREZL for treating intractable limb spasticity between October 2008 and August 2016 were recruited in the study. The candidates were individuals suffering from severe spasticity of the extremities caused by spinal cord pathology. All of them failed to respond to conventional treatment, including oral antispasmodic medications, botulinum toxin injection, physical therapy and application of orthotic devices. DLM consisted of two types of procedures, including Bischof \|| (dorsal longitudinal T) myelotomy ${ }^{14}$ (Figures 1 and 2a) and Pourpre (cruciate) myelotomy (Figure 2b). ${ }^{15}$ DLM and DREZL of the lumbosacral (L1 to S1) spinal cord segments were used for eliminating severe flexor spasm of both lower extremities and DREZL of the cervical (C5 to C8) spinal cord segments was performed for treating upper limb spasticity. This study was approved by our institutional review board and all applicable institutional regulations concerning the ethical use of human subjects were followed during the course of this research.

\begin{abstract}
Clinical evaluation
The Modified Ashworth Scale, ${ }^{16}$ Adductor Tone Rating Scale, ${ }^{17}$ Penn Spasm Frequency Scale, ${ }^{18}$ ambulatory status and existence of pressure ulcer were assessed before and after all operations. Mean MAS score was calculated from MAS score of all examined muscle groups, such as hip flexors, knee flexors and so on. In each patient, the number of examined muscle groups varied from 6 to 14 depending upon extent of operated cord segments. Mean ATRS and PSFS scores were calculated from ATRS and PSFS scores of all patients, respectively. In each patient, there was only one value of ATRS and PSFS scores. These parameters on the last follow-up were compared with the preoperative clinical assessment and were compared between procedures.
\end{abstract}

\section{Statistical analysis}

Data were analyzed using Statistical Package for the Social Sciences (SPSS) version 17.0 (SPSS Inc., Chicago, IL, USA). The MAS score, ATRS and PSFS before and after the operations were compared by using the paired samples $t$-test and Wilcoxon's signed-rank matched-pairs test. Comparison of clinical parameters between the operations was carried out by using the independent-sample $t$-test or Mann-Whitney U-test. Statistically significant level was indicated with $P<0.05$. 

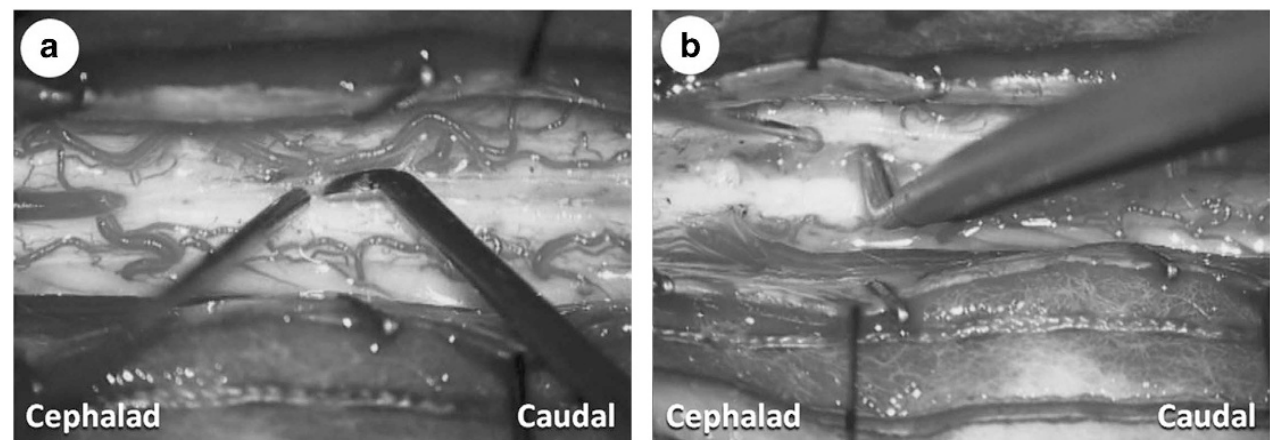

Figure 1. DLM by using Bischof II technique. (a) Midline myelotomy to the depth of the central canal. (b) Angled fine tips of bipolar coagulator are pointed to the lateral side.
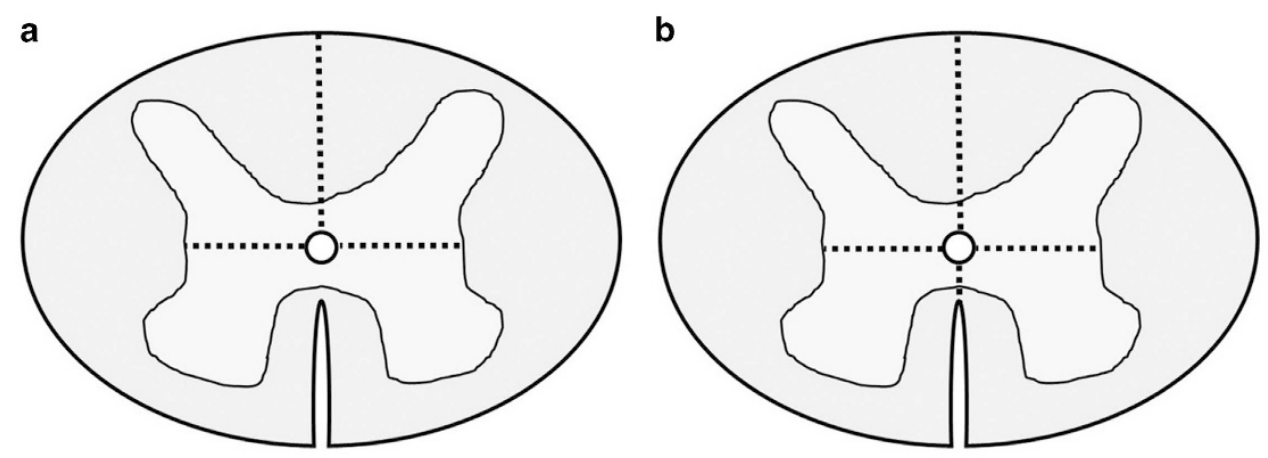

Figure 2. Axial view of the spinal cord showing two techniques in DLM. (a) Bischoff II myelotomy. Section of the midline structures to the depth of the central canal (sagittal dotted line) and disconnection between the ventral and dorsal horns bilaterally (coronal dotted line). (b) Pourpre myelotomy. Division of the spinal cord into two hemicords (sagittal dotted line) and bilateral disconnection, the ventral from the dorsal horns (coronal dotted line).
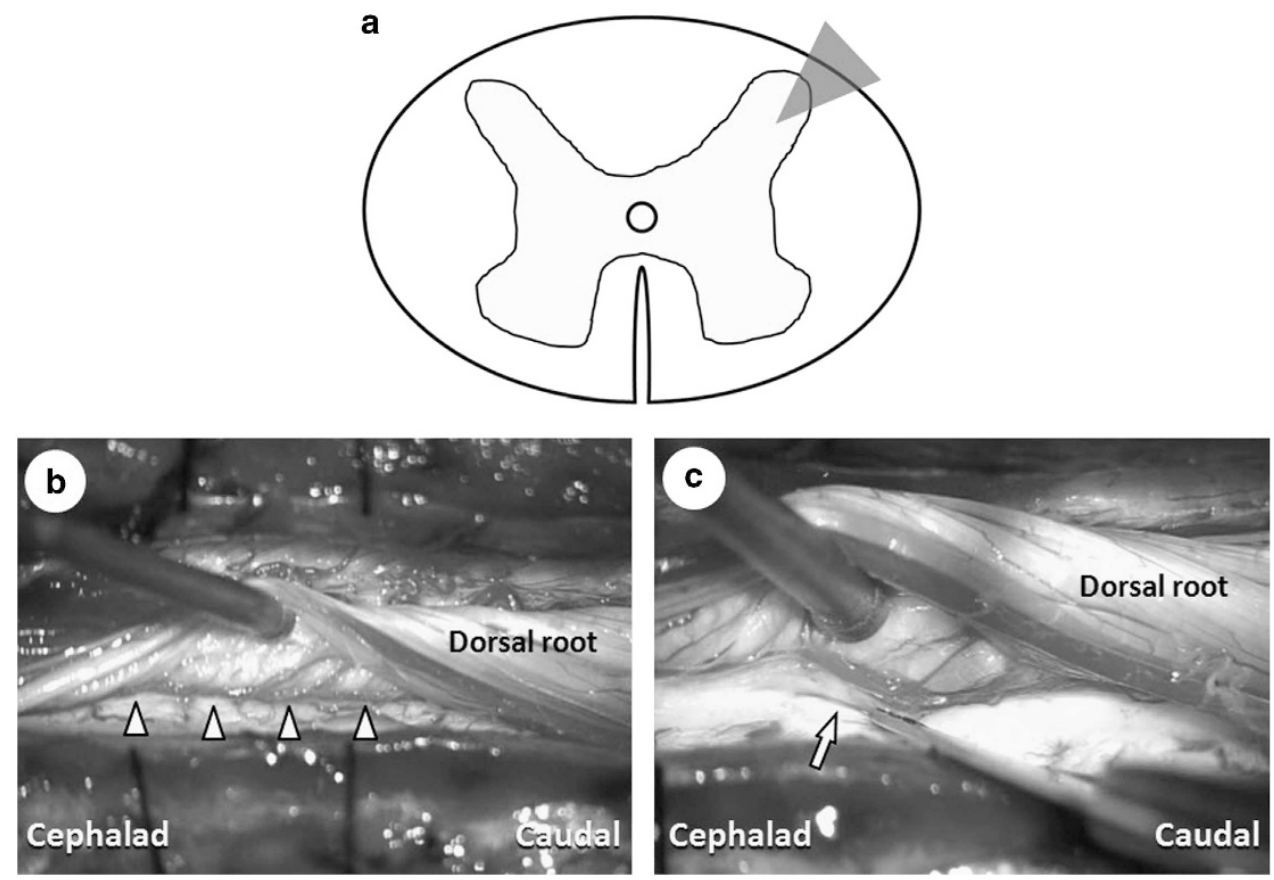

Figure 3. DREZL. (a) Axial view of the spinal cord showing area and trajectory of the lesioning (triangle). (b) Operative exposure of the dorsal root entry zone (arrowheads). (c) Longitudinal lesioning (arrow) of the dorsal root entry zone.

\section{RESULTS}

\section{Patients}

Of 18 patients, 12 were male and 6 were female. Mean age was $39.7 \pm 13$ years (range 18-65). Median duration of spasticity before surgery was 4 years (range 2-14). Spasticity was caused by spinal cord injury $(\mathrm{SCl})$ in 10 patients $(55.5 \%)$, spinal cord tumor in 3 (16.7\%), spinal tuberculosis in $2(11.1 \%)$ and other disorders (ruptured cavernoma, multiple sclerosis and disc herniation) in 


\begin{tabular}{|c|c|c|c|c|c|c|c|c|}
\hline 1 & M 45 & $\mathrm{SCl} / \mathrm{T} 8(\mathrm{~A})$ & Bischof II (L1-S1) & 73 & Unhealed & Healed & Bed-bound & Wheelchair \\
\hline 2 & M 57 & Ruptured cavernoma/T7 (A) & Bischof II (L1-S1) & 69 & - & - & Bed-bound & Wheelchair \\
\hline 3 & F 49 & MS/upper T (A) & Pourpre (L1-S1) & 63 & Unhealed & Healed & Bed-bound & Wheelchair \\
\hline 6 & M 18 & $\mathrm{SCl} / \mathrm{T} 6$ (A) & Bischof II (L1-S1) & 55 & Unhealed & Healed & Wheelchair & Wheelchair \\
\hline 7 & F 39 & $\mathrm{SCl} / \mathrm{C} 4-5(\mathrm{~A})$ & Bischof II (L1-S1) & 54 & Unhealed & Healed & Bed-bound & Wheelchair \\
\hline 8 & M 44 & Disc herniation/T7-8 (C) & DREZL (bilateral L1-S1) & 37 & - & - & Wheelchair & $\begin{array}{l}\text { Ambulator } \\
\text { with assistance }\end{array}$ \\
\hline 9 & M 25 & $\mathrm{SCl} / \mathrm{T} 4(\mathrm{~A})$ & Bischof II (L1-S1) & 35 & Unhealed & Healed & Wheelchair & Wheelchair \\
\hline 10 & M 41 & Spinal TB/T8 (A) & Bischof II (L1-S1) & 30 & Unhealed & Healed & Bed-bound & Wheelchair \\
\hline 11 & M 40 & $\mathrm{SCl} / \mathrm{C} 2(\mathrm{~A})$ & $\begin{array}{l}\text { DREZL (bilateral C5-C8, } \\
\text { L1-S1) }\end{array}$ & 28 & - & - & Bed-bound & Wheelchair \\
\hline 15 & M 26 & $\mathrm{SCl} / \mathrm{T} 10(\mathrm{~A})$ & Bischof II (L1-S1) & 14 & Unhealed & Healed & Wheelchair & Wheelchair \\
\hline 16 & M 31 & $\mathrm{SCl} / \mathrm{T} 7(\mathrm{~A})$ & Bischof II (L1-S1) & 11 & Unhealed & Improved & Wheelchair & Wheelchair \\
\hline 17 & F 65 & Spinal cord tumor/T8 (A) & Bischof II (L1-S1) & 8 & - & - & Wheelchair & Wheelchair \\
\hline 18 & M 52 & Spinal TB/T8 (C) & DREZL (bilateral L1-S1) & 6 & - & - & Bed-bound & Wheelchair \\
\hline
\end{tabular}

Abbreviations: -, none; AIS, American Spinal Injury Association Impairment Scale; Bischof II, Bischof II myelotomy; C, cervical; DREZL, dorsal root entry zone lesion; F, female; F/U, follow-up; L, lumbar; M, male; mo, months; MS, multiple sclerosis; postop, postoperative; Pourpre, Pourpre myelotomy; preop, preoperative; $\mathrm{S}$, sacral; $\mathrm{SCl}$, spinal cord injury; $\mathrm{T}$, thoracic; $\mathrm{TB}$, tuberculosis.

Table 2. Comparison of clinical parameters between before and after the procedures, and between DLM and DREZL

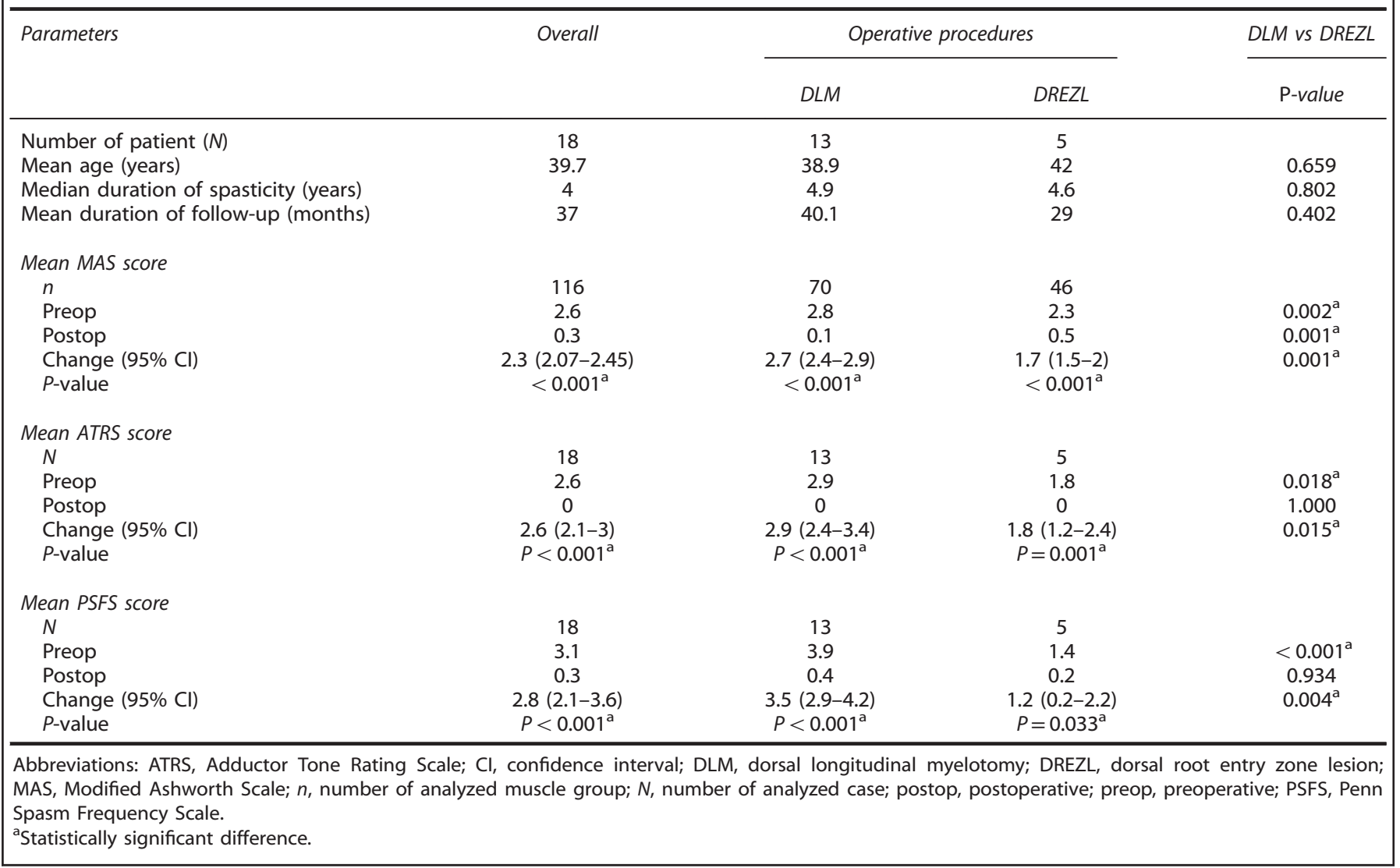



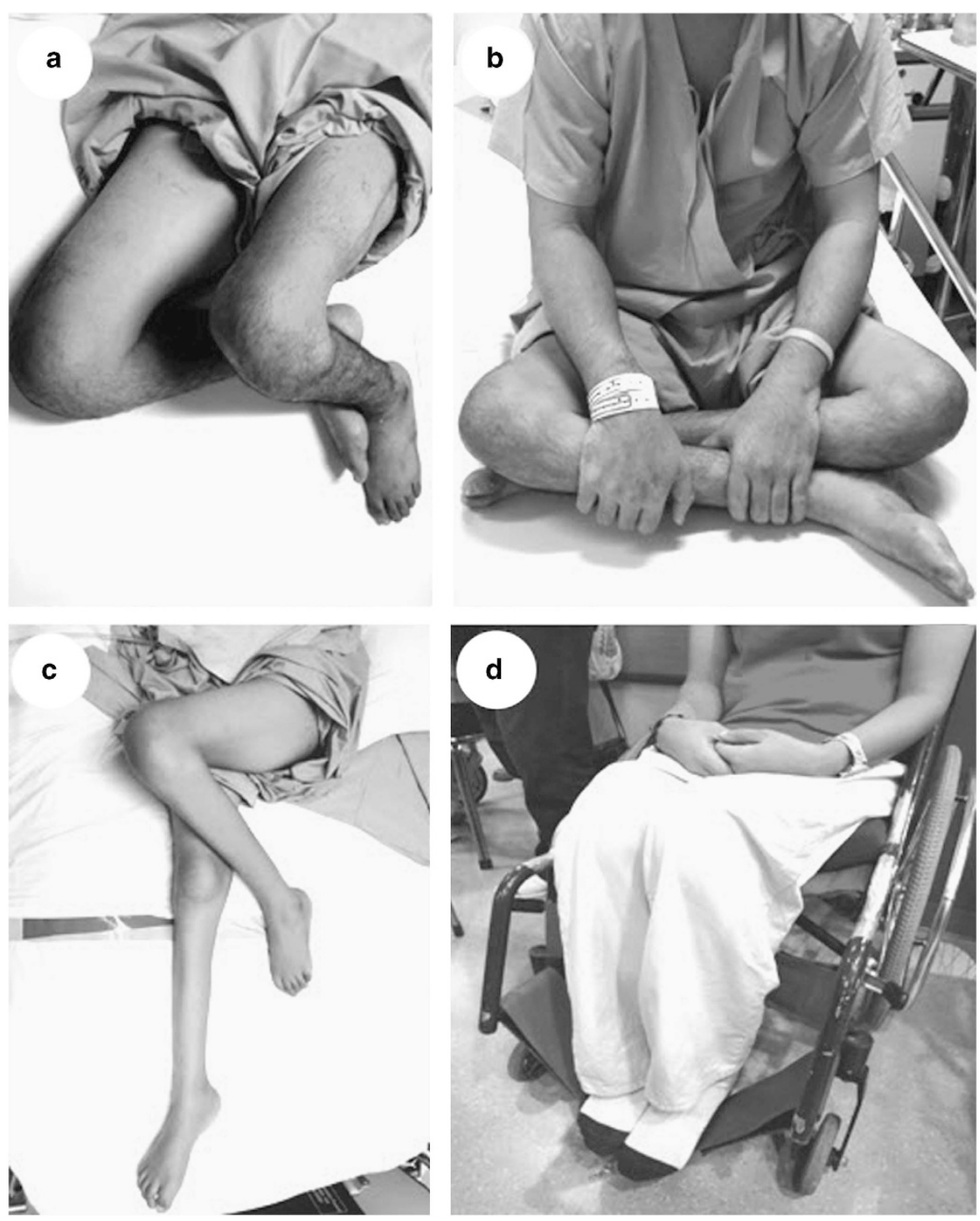

Figure 4. Improvement of sitting and ambulation after the procedures. (a) A complete thoracic SCl patient (case no. 1) suffered from severe flexor spasms of both legs before the surgery. (b) After Bischof II myelotomy on the L1-S1 spinal cord segments, spasticity was eliminated and the patient could sit better and ambulate with wheelchair. (c) A young female with incomplete thoracic $\mathrm{SCl}$ (case no. 5) caused by gunshot injury at the T10 spinal level. She had spastic paraplegia and intractable pain before the operation. (d) Following DREZL on the L1-S1 spinal cord segments, spasticity completely vanished and the pain markedly improved. Her pressure ulcer also healed.

3 (16.7\%). DLM and DREZL were performed in $13(72.2 \%)$ and 5 (27.8\%), respectively. Mean postoperative follow-up was $37 \pm 22$ months (range 6-73). Patient characteristics are documented in Table 1.

\section{Surgical outcomes}

Preoperatively, 12 patients had unhealed decubitus ulcers at the sacrococcygeal or trochanteric region. The ulcers were completely healed after surgical treatment of severe spasticity in 11 and improved in one. All of 7 patients who were bed-bound were able to return to use of a wheelchair postoperatively. One patient who was non-ambulatory before surgery (case no. 8) returned to an ambulatory status with assistance after DREZL.

Preoperative and postoperative clinical parameters are presented in Table 2. A statistically significant reduction of spasticity was present. ATRS and PSFS were significantly decreased in all operations and in overall analysis. In comparison between DLM and DREZL, the DLM group had preoperative mean MAS, ATRS and PSFS scores that were significantly higher than those of the DREZL group. Postoperatively, the mean MAS score of the DLM group was significantly lower than that of the DREZL group, whereas there was no difference in the mean ATRS and PSFS scores between both groups. The DLM group had a significantly greater change in mean MAS, ATRS and PSFS scores than the DREZL group.

Two patients in our series had concomitant intractable spasticity and severe pain. Case no. 3 presented with intractable painful flexor spasm of both lower limbs. Pourpre myelotomy provided satisfactory outcomes in this patient. Complete extinction of flexor spasms and complete pain relief were achieved. Case no. 5 had spastic paraplegia coexisting with post-SCI pain. Marked improvement of flexor spasms and a reduction of her average pain score (from 7 to 2) were accomplished after DREZL.

No recurrent spasticity was noted during the follow-up period.

\section{Complications}

One patient (case no. 4) developed hypotrophy of the bilateral quadriceps femoris several months following DLM. Because 14 of 
18 patients had complete spinal cord lesions (AIS A) and 1 was classified as AIS B, they required intermittent or permanent urinary catheterization and occasional fecal evacuation before surgery. All of these patients, including the remaining three with AIS C had no additional impairment of bowel and bladder function after surgery. There was no operative mortality, worsening of neurological status or development of spinal deformity in follow-up period. However, there was no evaluation of sexual function in this study.

\section{DISCUSSION}

Neurosurgery is an effective option in the treatment of intractable spasticity. In the past century, a wide variety of destructive surgeries on various neural structures had been commonly used. ${ }^{19,20}$ Over the recent years, with the development of an intrathecal drug delivery system, ITB has been increasingly utilized while the popularity of ablative surgery has gradually diminished. Nevertheless, in circumstances when ITB is unavailable or not affordable, lesioning procedures can be valuable in well-selected cases. Because these surgeries carry irreversible properties, careful preoperative evaluation for selecting surgical candidates is essential.

In the upper motor neuron syndrome, loss of supraspinal inhibitory control makes spinal motor neurons hyperexcitable and subsequently brings about exaggerated spinal reflexes., ${ }^{3,4}$ DLM and DREZL endeavor to suppress function of hyperactive reflex arcs. DLM disconnects the ventral and dorsal horns of the spinal cord ${ }^{13}$ whereas DREZL disrupts the spinal myotatic reflex at the level of dorsal root entry zone. ${ }^{21,22}$ Consequently, all procedures give rise to abolishment of spasticity. Localization of the precise levels of the spinal cord and collateral roots by utilizing reliable measures is mandatory to achieve favorable outcomes.

The Bischoff II myelotomy was pioneered by Bischoff in $1967 .{ }^{14}$ Several authors subsequently reported successful and reproducible results in eradication of intractable spasticity. ${ }^{23-27}$ In addition to coronal disconnection between the ventral and dorsal horns being the same as in Bischoff II myelotomy, the Pourpre procedure (cruciform myelotomy) divides the spinal cord into two halves. Theoretically, sagittal disconnection of the anterior white commissure disrupts the spinothalamic tract where it crosses the midline. Section of this tract which contains nociceptive fibers probably results in the superiority of Pourpre procedure to the Bischoff II myelotomy in the reduction of pain coinciding with spasticity. Furthermore, a comparative study revealed that patients who underwent the Pourpre myelotomy achieved significantly more pain reduction than those who underwent a Bischoff II myelotomy. ${ }^{27}$ These findings encouraged us to use the cruciform myelotomy in case no. 3 who had painful spastic triple flexion. Importantly, both types of DLM carry some risk of motor complications caused by injury to the lateral corticospinal tract or spinal motor neurons during lesioning with the low current microsurgical coagulator. We therefore perform DLM only in subjects without hope of regaining motor function and never offer this operation to patients with residual motor function. However, by using our lesioning technique, we have never encountered residual or recurrent spasticity as has been found in previous studies that used a myelotomy knife for lesioning. . $3,24,26,27^{2}$

DREZL not only interrupts spinal myotatic reflexes but also impedes nociceptive pathways within the spinal cord. ${ }^{21}$ The inhibition of both results in diminished spasticity and pain. Theoretically the surgery does not affect the descending motor pathways. Therefore, we use it in patients with residual motor function, with or without pain, and completely spastic limbs. DREZL preferentially reduces proximal than distal limb spasticity, so using two procedures, DREZL for proximal and selective peripheral neurotomy for distal spasticity, is sometimes required. $^{5,28}$ In our patients undergoing DREZL, results revealed a significant reduction of spasticity.

Eleven of 12 patients suffering from unhealed pressure ulcers had healed ulcers postoperatively. Spasticity affects posture and can lead to the development or worsening of pressure ulcers; conversely, pressure ulcers can aggravate spasticity resulting in a vicious cycle. Stopping severe spasticity can facilitate healing of decubitus ulcers. All of seven patients who were bedridden preoperatively could be positioned to sit, then were able to ambulate with a wheelchair (Figure 4), while another undergoing DREZL could return to walking with assistance. These outcomes imply that intractable lower limb spasticity prohibits patients from sitting, thus surgical correction can restore the potential to ambulate by wheelchair or walking.

In a comparison between DLM and DREZL, preoperative spasticity of the DLM group evaluated by the MAS, ATRS and PSFS scores was significantly more severe than that of the DREZL counterpart. The DLM group had the postoperative MAS scores and change in all of the three spasticity scores better than those of the DREZL group. These findings may indicate that the nature of spasticity in patients undergoing DLM tends to be more severe than in those undergoing DREZL in our study. We believe that DLM is superior to DREZL in annihilation of refractory spasticity. Moreover, selection of appropriate surgical methods can markedly reduce spasticity.

A major concern about spinal cord ablative procedures is additional neurological impairment, especially bowel, bladder and sexual dysfunction. Preoperatively, all of our patients with complete spinal cord lesions (AIS A) could not voluntarily control the urinary and anal sphincters, so sphincter function was not affected postoperatively. In patients with incomplete cord lesions (AIS B and C) who underwent DREZL, the procedure was performed carefully to avoid injury of the sacral spinal cord. By subjective assessment after surgery, there was no deterioration of bowel and bladder function reported by our patients. Preoperative and postoperative sexual functions were not studied in the present series. In our point of view, patients with voluntary control of the sphincters or still have active sexual function should not undergo destructive procedure on the spinal cord. The procedure may impair sphincter control and may result in erectile dysfunction in men or sexual dysfunction in female. These issues must be discussed with patients and documented before surgery.

\section{CONCLUSION}

Ablative neurosurgery on the spinal cord still has a major role in the treatment of widespread or regional spasticity, particularly in situations when ITB is unavailable. Localization of precise neural segments is crucial in the accomplishment of good outcomes. DLM and DREZL can effectively reduce spasticity, disrupt hyperactive pathologic reflexes, facilitate healing of pressure ulcers and improve the ability to ambulate. Nevertheless, because of the irreversible properties and potential neurologic complications of these surgeries, surgical candidates must be selected with caution.

\section{COMPETING INTERESTS}

The authors declare no conflict of interest.

\section{REFERENCES}

1 Dajpratham P, Kuptniratsaikul V, Kovindha A, Kuptniratsaikul PS, Dejnuntarat K. Prevalence and management of poststroke spasticity in Thai stroke patients: a multicenter study. J Med Assoc Thai 2009; 92: 1354-1360.

2 Charles PD, Gill CE, Taylor HM, Putman MS, Blair CR, Roberts AG et al. Spasticity treatment facilitates direct care delivery for adults with profound intellectual disability. Mov Disord 2010; 25: 466-473. 
3 Tabtimsuwan S, Sitthinamsuwan B, Chankaew E. Spasticity: a comprehensive review. Siriraj Med J 2011; 63: 32-37.

4 Kheder A, Nair KP. Spasticity: pathophysiology, evaluation and management. Pract Neurol 2012; 12: 289-298.

5 Sindou MP, Mertens P. Neurosurgery for spasticity. Stereotact Funct Neurosurg 2000; 74: 217-221.

6 Lazorthes Y, Sallerin-Caute B, Verdie JC, Bastide R, Carillo JP. Chronic intrathecal baclofen administration for control of severe spasticity. J Neurosurg 1990; 72: 393-402.

7 Azouvi P, Mane M, Thiebaut JB, Denys P, Remy-Neris O, Bussel B. Intrathecal baclofen administration for control of severe spinal spasticity: functional improvement and long-term follow-up. Arch Phys Med Rehabil 1996; 77: 35-39.

8 Zahavi A, Geertzen JH, Middel B, Staal M, Rietman JS. Long term effect (more than five years) of intrathecal baclofen on impairment, disability, and quality of life in patients with severe spasticity of spinal origin. $J$ Neurol Neurosurg Psychiatry 2004; 75: 1553-1557.

9 Boviatsis EJ, Kouyialis AT, Korfias S, Sakas DE. Functional outcome of intrathecal baclofen administration for severe spasticity. Clin Neurol Neurosurg 2005; 107: 289-295.

10 Natale M, Mirone G, Rotondo M, Moraci A. Intrathecal baclofen therapy for severe spasticity: analysis on a series of 112 consecutive patients and future prospectives. Clin Neurol Neurosurg 2012; 114: 321-325.

11 Varhabhatla NC, Zuo Z. Rising complication rates after intrathecal catheter and pump placement in the pediatric population: analysis of national data between 1997 and 2006. Pain Physician 2012; 15: 65-74.

12 Haranhalli N, Anand D, Wisoff JH, Harter DH, Weiner HL, Blate M et al. Intrathecal baclofen therapy: complication avoidance and management. Childs Nerv Syst 2011; 27: 421-427.

13 Sitthinamsuwan B, Nunta-Aree S, Nitising A, Tabtimsuwan S. The neurosurgical treatment of spasticity-an overview. Neurol Surg 2010; 1: 44-60.

14 Bischof W. On dorsal longitudinal myelotomy. Zentralbl Neurochir 1967; 28: 123-126.
15 Pourpre $\mathrm{H}$. Neurosurgical treatment of contractures in patients with posttraumatic paraplegia. Neurochirurgie 1960; 6: 229-236.

16 Bohannon RW, Smith MB. Interrater reliability of a modified Ashworth scale of muscle spasticity. Phys Ther 1987; 67: 206-207.

17 Snow BJ, Tsui JK, Bhatt MH, Varelas M, Hashimoto SA, Calne DB. Treatment of spasticity with botulinum toxin: a double-blind study. Ann Neurol 1990; 28: 512-515

18 Penn RD, Savoy SM, Corcos D, Latash M, Gottlieb G, Parke B et al. Intrathecal baclofen for severe spinal spasticity. N Engl J Med 1989; 320: 1517-1521.

19 Boop FA. Evolution of the neurosurgical management of spasticity. J Child Neurol 2001; 16: 54-57.

20 Sindou MP. History of the neurosurgical treatment of spasticity. Oper Tech Neurosurg 2005; 7: 96-99.

21 Sindou MP, Mertens P. Surgery in the dorsal root entry zone for spasticity in adults. Oper Tech Neurosurg 2005; 7: 157-162.

22 Sitthinamsuwan B, Phonwijit L, Ploypetch T. Neurosurgical management for cerebral palsy_part I: neuroablation. J Thai Rehabil Med 2011; 21: 73-84.

23 Laitinen L, Singounas E. Longitudinal myelotomy in the treatment of spasticity of the legs. J Neurosurg 1971; 35: 536-540.

24 Laha RK, Dujovny M, Osgood CP. Dorsal longitudinal myelotomy. Paraplegia 1976; 14: 189-194.

25 Yamada S, Perot PL Jr, Ducker TB, Lockard I. Myelotomy for control of mass spasms in paraplegia. J Neurosurg 1976; 45: 683-691.

26 Putty TK, Shapiro SA. Efficacy of dorsal longitudinal myelotomy in treating spinal spasticity: a review of 20 cases. J Neurosurg 1991; 75: 397-401.

27 Livshits A, Rappaport ZH, Livshits V, Gepstein R. Surgical treatment of painful spasticity after spinal cord injury. Spinal Cord 2002; 40: 161-166.

28 Sitthinamsuwan B, Chanvanitkulchai K, Nunta-aree S, Kumthornthip W, Pisarnpong A, Ploypetch T. Combined ablative neurosurgical procedures in a patient with mixed spastic and dystonic cerebral palsy. Stereotact Funct Neurosurg 2010; 88: 187-192. 